\title{
DIAGNÓSTICOS DE ENFERMAGEM DO PACIENTE NO PERÍODO PÓS-OPERATÓRIO IMEDIATO
}

\author{
NURSING DIAGNOSES OF THE PATIENT IN THE \\ IMMEDIATE POST-OPERATIVE PERIOD
}

\author{
Lidia Aparecida Rossi* \\ Fernanda Gaspar Torrati** \\ Emilia Campos de Carvalho*** \\ Alessandra Manfrim**** \\ Dulce Ferreira da Silva*****
}

ROSSI, L. A. et al. Diagnósticos de Enfermagem do paciente no período pós-operatório imediato. Rev.Esc.Enf.USP, v. 34, n. 2, p. 154-64, jun. 2000.

\section{RESUMO}

O objetivo deste estudo foi identificar os diagnósticos de enfermagem mais freqüentes de pacientes em pós-operatório imediato. Foi elaborada uma ficha de avaliação pós-anestésica do periodo pós-operatório que foi utilizada em 28 pacientes nas primeiras duas horas em que permaneceram em centro de recuperação pós-anestésica. Os diagnósticos de enfermagem foram estabelecidos com base na Taxionomia I dos Diagnósticos de Enfermagem da North American Nursing Diagnosis Association - NANDA. Foram identificados os seguintes diagnósticos: risco para injúria (100,0\%), risco para infecção (92,8\%), senso-percepção alterada (89,2\%), risco para aspiração (82,1\%), mobilidade física prejudicada (71,4\%), integridade tissular prejudicada (82,1\%) e hipotermia $(67,8 \%)$.

PALAVRAS-CHAVE: Diagnóstico de enfermagem. Cuidados de enfermagem. Cuidados pós-operatórios.

\section{ABSTRACT}

The aim of this study was to identify the nursing diagnoses that are more frequent in patients in immediate post-operative period. Data were collected and recorded on specially cards, through evaluation of 28 patients in the first two hours after the admission of the patient. The nursing diagnoses were set by one of the authors, based on Taxonomy I, proposed by North American Nursing Diagnosis Association - NANDA. The diagnoses that occurred mostly were: Risk for injury (100,0\%), Risk for infection (92,8\%), Sensory perceptual alterations (89,2\%), Risk for aspiration (82,1\%), Impaired physical mobility (71,4\%), Impaired tissue integrity (82,1\%) and Hypothermia (67,8\%).

KEYWORDS: Nursing diagnosis. Nursing care. Postoperative care.

\section{INTRODUÇÃO}

A assistência de enfermagem à pacientes no período pós-anestésico deve ter como objetivo garantir uma recuperação segura, prevenindo, detectando e atendendo às complicações que possam advir do ato anestésico cirúrgico. Embora o alcance desse objetivo esteja relacionado às situações que envolvem o paciente no período perioperatório como um todo, cabe ao centro de recuperação pós anestésica reunir recursos suficientes que assegurem a qualidade da assistência de enfermagem no período pós operatório imediato. A determinação da qualidade e da quantidade de recursos materiais ou

\footnotetext{
* $\quad$ Enfermeira, Professora Doutora do Departamento de Enfermagem Geral e Especializada da Escola de Enfermagem de Ribeirão Preto (EERP, USP).

** $\quad$ Enfermeira da Seção de Enfermagem de Recuperação do Hospital das Clínicas da Faculdade de Medicina de Ribeirão Preto

*** (HCFMRP). Enfermeira, Professora Titular do Departamento de Enfermagem Geral e Especializada EERP.

**** Enfermeira do Centro Cirúrgico do HCFMRP.

***** Enfermeira Chefe da Seção de Enfermagem de Recuperação do HCFMRP.
} 
humanos ou de ambos depende do objetivo do serviço e do perfil da clientela a ser atendida. Essa avaliação tem sido tradicionalmente realizada pelos enfermeiros com base em estatísticas e informações médicas, tais como: número de procedimentos de enfermagem e de leitos, média de permanência dos pacientes no centro de recuperação, porte e tipo de procedimento cirúrgico realizado. Acrescenta-se a essa situação o fato de que muitas vezes as intervenções de enfermagem são propostas com base em diagnósticos médicos e estabelecidas de forma genérica sem considerar a individualidade do paciente.

A utilização da taxionomia da NORTH AMERICAN NURSING DIAGNOSIS ASSOCIATION (NANDA, 1994) prevê a adoção de uma nova forma de comunicação na enfermagem e ainda um novo foco de atuação para o enfermeiro, seja no ensino, na pesquisa ou na assistência. O perfil diagnóstico dos pacientes, elaborado com base nessa taxionomia, oferece uma fundamentação para determinar as intervenções de enfermagem facilitando a implementação do planejamento da assistência de enfermagem. Assim, é possivel avaliar a necessidade de recursos e a qualidade da assistência da enfermagem e, a partir dessa avaliação, propor medidas para modificação da prática através de programas de educação continuada.

Na literatura, encontramos poucos estudos que identificaram o perfil diagnóstico de pacientes em pós-operatório imediato. NULL et al. (1995) propõem um instrumento de registro e coleta de dados que poderia ser utilizado no planejamento da assistência de enfermagem com base em diagnósticos de enfermagem que consideraram mais comuns e relevantes no periodo perioperatório. CIRILLO; HARDY (1991) desenvolveram um sistema de registro de informações de pacientes em pós operatório imediato utilizando os diagnósticos de enfermagem. Outros autores abordam a assistência de enfermagem em situações específicas no período pós-operatório como as complicações que envolvem as vias aéreas (ODOM, 1993) ou o sistema cardiovascular (BINES; LANDRON, 1993), mas não se fundamentam na taxionomia da NANDA. Já SUMMERS (1992), fazendo referência à taxionomia da NANDA, estudou a alteração do nível de consciência dos pacientes em pós-operatório imediato.

Diante do exposto, consideramos relevante a realização de um estudo com o objetivo de identificar os diagnósticos de enfermagem mais freqüentes no pós operatório imediato de pacientes internados em centro de recuperação pós-anestésica.

\section{METODOLOGIA}

\author{
O estudo foi realizado no Centro de
}

Recuperação Pós-anestésica (CR) do Hospital das
Clínicas da Faculdade de Medicina de Ribeirão Preto - HCFMRP-USP, após aprovação do Comitê de Ética e Pesquisa da Instituição. O CR desse hospital conta com 20 leitos, recebendo em média, 30 pacientes por dia, de diferentes especialidades. Fizeram parte da amostra os pacientes adultos, nas primeiras duas horas de permanência no Centro de Recuperação submetidos a cirurgias das seguintes especialidades: cirurgia geral, ortopedia, ginecologia, oftalmologia, otorrinolaringologia, cabeça e pescoço, vascular e neurocirurgia; e que receberam diferentes tipos de anestesia geral e regional. Os dados foram coletados por um período de dois meses, por 20 alunos do 30 ano de graduação de enfermagem, quando estavam cursando a disciplina Enfermagem Cirúrgica, durante o período de estágio no $\mathrm{CR}$, por uma enfermeira da Unidade e por uma das autoras deste estudo. Todos os dados foram validados por esta autora na medida em que eram coletados.

Como instrumento de coleta de dados foi utilizada uma Ficha de avaliação pós anestésica do período pós-operatório imediato (Anexo). Esse instrumento foi elaborado com base nas considerações de DOENGES et al. (1993), SMELTZER; BARE (1993), MONAHAN et al. (1994) e NULL et al. (1995) e na experiência dos enfermeiros que atuam nessa Unidade. $\mathrm{O}$ instrumento foi testado conforme proposto por POLIT ; HUNGLER (1995), sendo submetido à apreciação de cinco enfermeiros que atuam nessa Unidade e modificado atendendo às sugestões feitas no pré-teste. A coleta de dados foi realizada conforme os passos adotados no serviço: na primeira hora após a admissão do paciente a cada 15 minutos e na segunda hora a cada 30 minutos. Dependendo do estado do paciente, esses intervalos de tempo foram diminuídos e esta situação foi registrada. Foi solicitado também aos 20 alunos e a enfermeira que coletaram os dados a avaliação do instrumento. Após a coleta dos dados, foram estabelecidos os diagnósticos de enfermagem, por uma das autoras do estudo, com base na taxionomia dos diagnósticos de enfermagem apresentada por NÓBREGA; GARCIA (1994), substituindo-se o termo potencial por risco conforme apresentado pela NANDA (1994). Para o estabelecimento dos diagnósticos utilizou-se o processo de raciocínio diagnóstico proposto por RISNER (1986) e aplicado por CARVALHO; JESUS (1997).

\section{RESULTADOS E DISCUSSÃO}

Foram avaliados 28 pacientes, com idades superiores a 12 anos, admitidos no CR nos meses de setembro e outubro de 1997. Os pacientes foram submetidos a diferentes tipos de cirurgias e receberam diferentes tipos de anestesia conforme mencionado na Metodologia. 
O instrumento utilizado para coleta e registro de dados permitiu a identificação de 16 diferentes categorias diagnosticas que estão apresentadas na Tabela 1. Todas as categorias diagnósticas foram estabelecidas com base nas informações apresentadas no instrumento que foi considerado completo pelos alunos e pela enfermeira que participaram da coleta de dados. Entretanto, consideramos importante incluir neste impresso informações sobre a classificação

\section{Diagnósticos de enfermagem mais freqüentes}

das cirurgias realizadas quanto ao potencial de contaminação. Um aspecto apontado pelos alunos de graduação foi o espaço para realização dos registros, que foi considerado limitado. Em função desta situação, algumas anotações foram realizadas nas margens do instrumento. Os alunos consideraram que o instrumento auxiliou no processo de coleta de dados e permitiu a reunião de informações sobre o paciente em um único impresso.

Tabela 1 - Distribuição das categorias diagnósticas identificadas em pacientes em pós operatório imediato. Ribeirão Preto, 1997.

\begin{tabular}{|c|c|c|}
\hline \multirow[t]{2}{*}{ Categoria diagnostica } & \multicolumn{2}{|c|}{ Freqüência } \\
\hline & $\mathrm{N}$. & $\%$ \\
\hline Risco para Injúria & 28 & 100,0 \\
\hline Risco para Infecção & 26 & 92,8 \\
\hline Senso-percepção Alterada & 25 & 89,2 \\
\hline Risco para Aspiração & 23 & 82,1 \\
\hline Integridade da Tissular Prejudicada & 23 & 82,1 \\
\hline Mobilidade Física Prejudicada & 20 & 71,4 \\
\hline H i potermia & 19 & 67,8 \\
\hline Dor & 11 & 32,2 \\
\hline Padrão Respiratório Ineficaz & 06 & 21,4 \\
\hline Risco para Alteração na Perfusão Tissular & 03 & 10,7 \\
\hline Integridade da Pele Prejudicada & 03 & 10,7 \\
\hline Débito Cardíaco Diminuído & 02 & 7,1 \\
\hline Medo & 02 & 7,1 \\
\hline Déficit de Volume de Líquidos & 01 & 7,1 \\
\hline Risco para Déficit de Volume de Líquidos & 01 & 3,5 \\
\hline Alteração na Eliminação Urinária & 01 & 3,5 \\
\hline
\end{tabular}

Com exceção dos diagnósticos de medo e senso-percepção alterada, os diagnósticos apresentados na Tabela 1 são apontados na literatura, por diferentes autores, como possiveis diagnósticos de enfermagem para pacientes em período pós-operatório imediato (SMELTZER ; BARE, 1993; PHIPPS et al., 1994 e MONAHAN et al., 1994). Esses autores acrescentam ainda o diagnóstico de ansiedade como um possível diagnóstico para o paciente no período pósoperatório imediato. Neste estudo, esse diagnóstico não foi observado; entretanto, foi identificado o diagnóstico de medo em dois pacientes $(7,1,0 \%)$ e, nos dois casos foi relacionado ao desconhecimento dos efeitos da anestesia, embora na Taxionomia da NANDA (1994) esses fatores necessitem ainda ser desenvolvidos. A identificação do objeto de medo foi verbalizada pelos pacientes como "receio de dormir e não acordar mais". A presença deste diagnóstico de enfermagem no pós-operatório imediato pode ser prevenida ou minimizada no período pré-operatório através de medidas educativas sobre os procedimentos 
anestésicos e cirúrgicos. Pesquisas têm indicado que, através da orientação ao paciente e à família no periodo pré-operatório, podem ser afastados muitos fatores de estresse psicológico no pós-operatório imediato (URSI ; MACUL, 1987; PANZA, 1977).

A dor é um diagnóstico muito comum no pós operatório imediato e pode resultar da incisão e da manipulação de tecidos e órgãos; por essa razão, embora este diagnóstico tenha sido identificado em apenas 11 dos 28 pacientes $(39,2 \%)$, consideramos importante mencioná-lo. No pós-operatório imediato a dor é mais freqüente nas cirurgias de grande porte das disciplinas torácicas, abdominais e ortopédicas (DRAIN, 1994). Todos os pacientes que referiram dor haviam sido submetidos a cirurgias de grande porte das seguintes especialidades: ginecologia, cirurgia vascular, ortopedia, cirurgia geral e neurocirurgia.

Quando o paciente refere dor no pós-operatório imediato é importante não atribuir a dor unicamente à incisão, mas tentar identificar as possiveis causas de dor. Para PHIPPS et al. (1994), além da incisão cirúrgica, a presença da dor pode estar relacionada a estimulação das terminações nervosas por substâncias químicas utilizadas durante a cirurgia, a isquemias causadas por interferência no suprimento de sangue para os tecidos, por pressão, espasmo muscular ou edema. Segundo DRAIN ; SHIPLEY (1981), a dor no paciente em pósoperatório imediato varia de acordo com o seu nível de consciência.

Um outro aspecto muito importante no Centro de Recuperação pós-anestésica é a avaliação do paciente quanto a função respiratória. Dos 28 pacientes avaliados, seis apresentaram o diagnóstico de padrão respiratório ineficaz. Segundo PHIPPS et al. (1994), dependendo da droga e da dosagem utilizada na anestesia, as alterações no padrão respiratório podem ocorrer a partir do momento em que a droga foi administrada até vários dias depois da intervenção cirúrgica.

Nas Tabela 2 e 3, a seguir, as categorias diagnósticas classificadas como reais e de risco, cuja freqüência foi superior a $60,0 \%$, estão apresentadas de acordo com os respectivos fatores relacionados e características diagnósticas. Consideramos essas categorias como as que apresentaram maior freqüência nos pacientes estudados.

Tabela 2 - Distribuição das categorias diagnósticas, caracterizadas como reais, que apresentaram freqüência acima de $60,0 \%$ de acordo com os fatores relacionados e características definidoras identificadas. Ribeirão Preto, 1997.

\begin{tabular}{lccll}
\hline \multicolumn{1}{c}{$\begin{array}{c}\text { Categorias } \\
\text { diagnósticas }\end{array}$} & Freqüência & \multicolumn{1}{c}{ Fatores relacionados } & Características Definidoras \\
& $\mathbf{N}^{\circ}$ & $\%$ & & \\
\hline $\begin{array}{l}\text { Senso- } \\
\text { percepção } \\
\text { alterada }\end{array}$ & 25 & 89,2 & $\begin{array}{l}\text { Alterações químicas } \\
\text { exógenas (secundária a } \\
\text { anestesia) }\end{array}$ & $\begin{array}{l}\text { Mudança verificada na acuidade } \\
\text { sensorial, padrões de comunicação } \\
\text { alterados. }\end{array}$
\end{tabular}

\begin{tabular}{|c|c|c|c|c|}
\hline $\begin{array}{l}\text { Integridade } \\
\text { tissular }\end{array}$ & 23 & 82,1 & $\begin{array}{l}\text { Fatores mecânicos } \\
\text { (procedimento cirúrgico) }\end{array}$ & $\begin{array}{l}\text { Tecido lesado ou destruído (córnea, } \\
\text { mucosas, pele ou subcutâneo). }\end{array}$ \\
\hline
\end{tabular}

tissular
Prejudicada

\begin{tabular}{|c|c|c|c|}
\hline $\begin{array}{l}\text { Mobilidade } \\
\text { Fisica } \\
\text { Prejudicada }\end{array}$ & 71,4 & $\begin{array}{l}\text { Força e resistência } \\
\text { diminuidas, dor ou } \\
\text { desconforto }\end{array}$ & $\begin{array}{l}\text { Restrição dos movimentos imposta por } \\
\text { prescrição médica, relutância em tentar } \\
\text { movimentar-se, inabilidade de } \\
\text { movimentação }\end{array}$ \\
\hline
\end{tabular}

\begin{tabular}{|c|c|c|c|c|}
\hline Hipotermia & 19 & 67,8 & $\begin{array}{l}\text { Exposição a ambiente frio, } \\
\text { metabolismo alterado } \\
\text { (secundário ao uso de } \\
\text { drogas anestésicas, idade, } \\
\text { vasodilatação) }\end{array}$ & $\begin{array}{l}\text { Redução da temperatura abaixo da } \\
\left.\text { faixa normal (menor que } 36^{\circ} \mathrm{C}\right), \\
\text { tremores e bradicardia. }\end{array}$ \\
\hline
\end{tabular}


O diagnóstico de senso-percepção, apresentado na Tabela 2, é definido pela NANDA (1994) como "o estado no qual o indivíduo experimenta uma mudança na quantidade ou tipo de estímulos percebidos, acompanhada por uma resposta diminuída, exagerada, distorcida ou enfraquecida a tais estimulos". MAcFARLAND; MAcFARLANE (1989) afirmam que a percepção sensorial é uma habilidade de receber e interpretar informações e que é um requisito para a cognição. Assim, considerando essas afirmações e as características definidoras apresentadas pelos pacientes, o diagnóstico de senso-percepção alterada foi identificado em 25 dos 28 pacientes estudados $(89,2 \%)$. NULL et al. (1995) também consideram o diagnóstico de senso-percepção alterada como possível para os pacientes em pós operatório imediato.

No Centro de Recuperação Pós-Anestésica, o paciente pode apresentar-se sonolento, confuso, com senso-percepção alterada. Com base na Taxionomia da NANDA, conforme apresentada por NÓBREGA; GARCIA (1994), podemos dizer que os fatores relacionados a esse diagnóstico apresentados pelos pacientes estudados foram: recepção, transmissão ou integração sensorial alterada, alteração química exógena secundária ao efeito residual da anestesia, ao tipo de anestésico utilizado e ao tipo de anestesia. $\mathrm{O}$ paciente que recebeu anestesia geral, por exemplo, usualmente apresenta redução do nível de consciência no período pós-operatório imediato. Esses pacientes geralmente despertam ao estímulo tátil ou verbal, ou ambos (ROTHROCK, 1990 e PHIPPS et al., 1994 ). ROTHROCK (1990) também afirma que essa alteração no nivel de consciência pode ter como conseqüência a mobilidade fisica prejudicada, risco para injúria, alteração no processo de pensamento e déficit de conhecimento.

Para DRAIN (1994), todos os agentes anestésicos afetam o sistema nervoso central e ainda não podemos dizer que conhecemos exatamente como a narcose ocorre, pois os anestésicos geralmente são depressores não seletivos. Os pacientes apresentaram mudança na percepção sensorial, apatia, mudança nas respostas usuais aos estímulos, caracterizada por estado de sonolência e mudança no padrão de comportamento, manifestada por agitação. Embora apresentassem esses sinais os pacientes identificavam o local onde estavam e demostravam ter conhecimentos sobre o término da cirurgia. Nesse sentido, é importante considerar que ao serem transferidos para o Centro de Recuperação, e logo após a sua chegada neste local, os pacientes são informados sobre o término da cirurgia e sobre sua localização.

A mobilidade física prejudicada foi identificada em 20 dos 28 pacientes avaliados $(71,4 \%)$. Os fatores relacionados a esse diagnóstico foram a força e resistência diminuídas, secundárias a anestesia, dor e desconforto. Esse diagnóstico é definido pela NANDA
(1994) como o estado no qual o indivíduo experimenta uma limitação na habilidade movimentos fisicos. Esse diagnóstico foi manifestado pela presença de restrição de movimentos impostos pela prescrição médica, de inabilidade de movimentação e relutância em tentar movimentar-se.

O diagnóstico de hipotermia foi firmado para 19 dos 28 pacientes $(67,8 \%)$ e foram identificados como fatores relacionados a exposição a ambiente frio, metabolismo alterado (secundário ao uso de drogas anestésicas, idade, vasodilatação) e como características definidoras desse diagnóstico: a temperatura abaixo da faixa normal (menos de $36 \mathrm{OC}$, apresentada por todos os pacientes que apresentaram esse diagnóstico), tremores e bradicardia apresentados por quatro pacientes. Segundo MEEKER; ROTHOROCK (1997), mais de 60,0\% dos pacientes apresentam hipotermia no período do pós-operatório imediato. O centro regulador da temperatura no cérebro é deprimido pelas drogas utilizadas na anestesia geral e as atividades musculares (tremores), que normalmente ajudam o corpo a gerar calor, são inibidas pelos relaxantes e narcóticos. Essa situação associada ao ambiente frio do Centro de Recuperação, a administração de infusões frias durante a cirurgia e à exposição da ferida cirúrgica ao ambiente frio da sala de cirurgia contribuem para o estado de hipotermia. A hipotermia produz vasoconstricção, diminui a freqüência cardíaca, aumenta a pressão sangüinea e eventualmente aumenta o trabalho cardiaco (PHIPPS et al., 1994).

Pacientes com extremos de idade e debilitados apresentam maiores riscos para alterações na temperatura no pós-operatório imediato. E importante esclarecer que neste estudo, a temperatura foi mensurada através da colocação do termômetro na axila e, como afirma DRAIN (1994), esta temperatura indica a temperatura da pele e não é uma medida precisa da temperatura do sangue. Dos 28 pacientes estudados quatro tinham idade acima de 60 anos e apresentaram hipotermia manifestada por temperatura abaixo de $36 \mathrm{0C}$, tremores e bradicardia.

A integridade tissular prejudicada foi identificada em 23 dos 28 pacientes $(82,1 \%)$. Os fatores, relacionados à esse diagnóstico foram identificados como fatores mecânicos, secundários ao procedimento cirúrgico; e as características definidoras foram: tecido lesado ou destruído (córnea, mucosas, pele ou subcutâneo). Este diagnóstico é definido pela NANDA (1994) "como o estado em que o indivíduo apresenta lesão em mucosas, córnea, tecido cutâneo ou subcutâneo". Durante a cirurgia, os pacientes também apresentam riscos para alteração na integridade da pele resultantes de determinadas posições cirúrgicas desconfortáveis por tempo prolongado ou da presença de fatores de risco como alterações no estado nutricional e idade avançada. No centro de 
Recuperação pós-anestésica a pele do paciente deve ser avaliada quanto a presença de possiveis traumatismos, tais como: queimaduras decorrentes do uso de bisturis elétricos, ferimentos provocados por posicionamento prolongado na mesa cirúrgica e pela falta de sensibilidade devido a anestesia.

Tabela 3 - Distribuição das categorias diagnósticas, caracterizadas como de risco ou potenciais, que apresentaram freqüência acima de 60,0\%, de acordo com os fatores de risco identificados. Ribeirão Preto, 1997.

\begin{tabular}{lccl}
\hline $\begin{array}{c}\text { Categorias } \\
\text { diagnósticas }\end{array}$ & Freqüência & \multicolumn{1}{c}{ Fatores de Riscos } \\
\hline Risco para Injúria & 28 & 100,0 & $\begin{array}{l}\text { Função bioquímica ou reguladora, (disfunção sensorial), } \\
\text { fatores físicos (lesão da pele, alterações na mobilidade) }\end{array}$ \\
\hline Risco para Infecção & 26 & 92,8 & $\begin{array}{l}\text { Defesa primária insuficiente (solução de continuidade da } \\
\text { pele, trauma tissular) e procedimentos invasivos }\end{array}$ \\
\hline Risco para Aspiração & 23 & 82,1 & $\begin{array}{l}\text { Situações que dificultam a elevação da parte superior do corpo, } \\
\text { diminuição do nivel de consciência, depressão dos reflexos da } \\
\text { tosse e deglutição, aumento da pressão intragástrica, o } \\
\text { vômito)administração de medicamentos (drogas que } \\
\text { potencializam o Vômito) }\end{array}$ \\
\hline
\end{tabular}

O diagnóstico de risco para infecção no paciente em pós-operatório imediato está relacionado aos procedimentos invasivos e à defesa primária insuficiente provocada pela solução de continuidade da pele e ao trauma tissular. Neste estudo, 26 dos 28 pacientes $(92,8 \%)$ apresentaram o diagnóstico de risco para infecção. A infecção de ferida cirúrgica é uma complicação séria que interfere no processo de cicatrização e pode aumentar o desconforto do paciente. As primeiras 24 a 48 horas depois da cirurgia são críticas, porque o processo de inflamação se inicia para destruir bactérias que possam ter sido depositadas enquanto a ferida estava aberta. Um aspecto a ser considerado neste diagnóstico é a classificação da cirurgia quanto ao grau de contaminação além de outros fatores tais como idade, presença de doenças crônicas, hábitos, estado nutricional e metabólico, que fornecerão uma idéia do risco de infecção da ferida a que cada paciente está exposto (PHIPPS et al., 1994). Assim, decidimos incluir no instrumento utilizado para coleta e registo dos dados a classificação da cirurgia quanto ao grau de contaminação.

As intervenções de enfermagem no período trans-operatório não podem mudar esses fatores, mas tem como objetivo o controle do número e do tipo de microorganismos durante a cirurgia. $\mathrm{O}$ diagnóstico de risco de infecção também pode estar relacionado com os locais de infusão que devem ser observados quanto a permeabilidade, coloração da pele e temperatura. Outros aspectos devem ser observados na ferida cirúrgica visando a prevenção de infecção e o favorecimento do processo de cicatrização, a saber: presença de hematomas, coloração da pele, temperatura, e manutenção do curativo seco e limpo.

O diagnóstico de risco para injúria é definido pela NANDA (1994) "como o estado no qual o indivíduo apresenta risco de injúria resultante da interação das condições ambientais com os recursos adaptativos defensivos". Esse diagnóstico foi identificado em 28 pacientes $(100,0 \%)$ e foram identificados como fatores de risco: estado de agitação, diminuição do nível de consciência e diminuição das respostas à estímulos internos e externos. O paciente com diagnóstico de senso-percepção alterada deve ser protegido de posições inadequadas que poderão causar desconforto e lesões da pele. Nesse sentido é preciso tomar cuidados especiais com lâmpadas aquecidas e bolsas de água quente para prevenir queimaduras. A aspiração é uma das complicações respiratórias que podem ocorrer no pós-operatório imediato. O paciente que está com senso-percepção alterada, dificuldade de elevar a parte superior do corpo e de lateralizar a cabeça pode aspirar o conteúdo gástrico no pósoperatório imediato. As conseqüências dessa situação podem ser desastrosas para o paciente, pois a aspiração do conteúdo gástrico pode provocar irritação, pneumonias, destruição da mucosa traqueal e aumento do risco de infecção.

Neste estudo, 21 dos 28 pacientes $(82,1 \%)$ apresentaram diagnóstico de risco para aspiração. Os fatores de risco identificados como relacionados a esse diagnóstico foram: dificuldade de elevar parte superior do corpo, depressão dos reflexos da tosse e deglutição, aumento da pressão intragástrica, 
administração de medicamentos (drogas anestésicas que potencializam o vômito). DRAIN; SHIPLEY (1981) salientam que as diferentes drogas utilizadas na anestesia também podem causar náusea e vômito, o que aumenta o risco de aspiração.

\section{CONSIDERAÇÕES FINAIS}

O instrumento utilizado para coleta e registro de dados permitiu a identificação de 16 diferentes diagnósticos. Todos os diagnósticos foram estabelecidos com base nas informações apresentadas no instrumento que foi considerado completo pelos alunos e pela enfermeira que coletou os dados. Embora tenham sugerido uma melhor distribuição dos espaços para registro, os alunos consideraram que o instrumento auxiliou no processo de coleta de dados e permitiu a reunião de informações sobre o paciente em um único impresso.

Os pacientes em pós-operatório imediato apresentaram como diagnósticos mais freqüentes: risco para injúria, risco para infecção, senso-percepção alterada, risco para aspiração, mobilidade física prejudicada, integridade tissular prejudicada e hipotermia. Os diagnósticos identificados nesse estudo enfocam prioritariamente os aspectos biológicos da assistência ao paciente. É provável que isto tenha ocorrido porque neste momento grande parte dos pacientes apresentaram o diagnóstico de sensopercepção alterada $(89,2 \%)$ e assim, têm dificuldades de expressar sentimentos e emoções. Esses resultados podem fundamentar as intervenções de enfermagem a serem planejadas para pacientes em pós-operatório imediato e também o ensino de graduação e de profissionais de enfermagem. A identificação da freqüência dos diagnósticos em populações específicas pode constituir uma base importante para o planejamento de recursos humanos e materiais visando a melhora da qualidade da assistência de enfermagem.

\section{REFERÊNCIAS BIBLIOGRÁFICAS}

BINES, A. S.; LANDRON, S L Cardiovascular emergencies in the post anesthesia care unit. Nurs. Clin. North Am., v. 28, n. 3, p.493-505, 1993.

CARVALHO, E. C. de; JESUS, C. A. C. Raciocínio clínico na formulação do diagnóstico de enfermagem para o indivíduo. In: ANTUNES, M. J.; SilvA, M. T. N. (org.). O uso do diagnóstico na prática de enfermagem. Brasília, ABEn, 1997. p. 27-38.

CIRILLO, B. I.; HARDY, E. Incorporating nursing diagnosis in postanesthesia care unit record. J. Post Anesth. Nurs., v. 6, n. 5 , p.336-41; 1991.
DOENGES, M. E. et al. Nursing care plans: guidelines for planning and documenting patient care. Philadelphia, F A Davis, 1993.

DRAIN, C. B. The post anesthesia care unit: a critical care approach to post anesthesia nursing. Philadelphia, W.B. Sunders, 1994.

SHIPLEY, S. P. Enfermagem na sala de recuperação. Rio de Janeiro, Interamericana, 1981.

McFARLAND, G. K.; McFARLENE, E. A. Nursing diagnosis \& intervention: planning for patient care. St Louis, Mosby, 1989.

MEEKER, M. H.; ROTHOROCK, J. C. Alexander: cuidados de enfermagem ao paciente cirúrgico. Rio de Janeiro, Guanabara Koogan, 1997

MONAHAN, F. D. et al. Nursing care of adults. Philadelphia, Saunders, 1994.

NORTH AMERICAN NURSING DIAGNOSIS ASSOCIATION (NANDA)- Nursing diagnoses: definitions \& classification. St Louis, NANDA, 1994.

NÓBREGA, M. L. da ; GARCIA, T. R. (Org.) Uniformização da linguagem dos diagnósticos de enfermagem da NANDA: sistematização das propostas do II SNDE. João Pessoa, CNDRE/ GIDE - PB, 1994.

NULL, S. et al. Development of a perioperative Nursing Diagnoses flow sheet. AORN J., v. 61, n. 3, p. 547-57, 1995.

ODOM, J. L. Airway emergencies in the post anesthesia care unit. Nurs.Clin. North Am., v. 28, n. 3, p.483-91, 1993.

PANZA, A. M. M. Efeito da visita pré operatória da enfermeira do centro cirúrgico sobre o estresse do paciente no pré-operatório e no pós-operatório. São Paulo, 1977. 75p. Dissertação (Mestrado) - Escola de Enfermagem, Universidade de São Paulo.

PHIPPS, W. J. et al. Medical surgical nursing: concepts and clinical practice, St Louis, Mosby, 1994.

POLIT, D. ; HUNGLER, B. P. Fundamentos de pesquisa em enfermagem. Porto Alegre, Artes Médicas Sul, 1995.

RISNER, P. B. Diagnosis: analysis and sinthesis of data. In: CHRISTENSEN, P.J.; KENNEY, J. W. Nursing process: conceptual models. 4. ed. St. Louis, Mosby, 1986. p 124-50.

ROTHROCK, J. C. Perioperative nursing care planning. St. Louis, Mosby, 1990.

SMELTZER, S. C. ; BARE, B. G. Brunner/Suddarth tratado de enfermagem médico-cirurgica. 7.ed. Rio de Janeiro, Guanabara Koogan , 1993.

SUMMERS, S. Concept analysis methodology: applications to altered level of consciousness. J Post Anesth Nurs., v. 7, n. 4, p.273-7, 1992.

URSI, E. S.; MACUL, S. F. A atuação do enfermeiro de centro cirúrgico em visitas pré e pós-operatórias. Enfoque, v. 15, n. 1, p. 4-6, 1987. 


\section{FICHA DE AVALIAÇÃO PÓS-ANESTÉSICA DO PÓS-OPERATÓRIO IMEDIATO}

\section{I - INFORMAÇÕES GERAIS:}

- Cirurgia proposta

- Cirurgia realizada.

- Anestesia

- Complicações.

- Data e horário de chegada no SER

• Data e horário de saída do SER

\section{II - FATORES QUE AFETAM 0 RISCO CIRÚRGICO:}

Tabagismo ( )

Diabetes ( )

Uso de drogas ( ) Alcoolismo ( ) Uso de medicações ( ); especificar ....

Insuficiência Cardíaca Congestiva ( ) Hipertensão Arterial ( ) Alergias ( ) especificar

Doenças respiratórias ( ) Estado nutricional (especificar).

.....; idade.

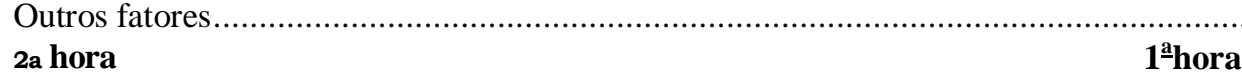

1-hor
30'

$45^{\prime}$

$60 '$

$90^{\prime}$

$120^{\prime}$

III - FUN ÃO RESPIRATÓRIA

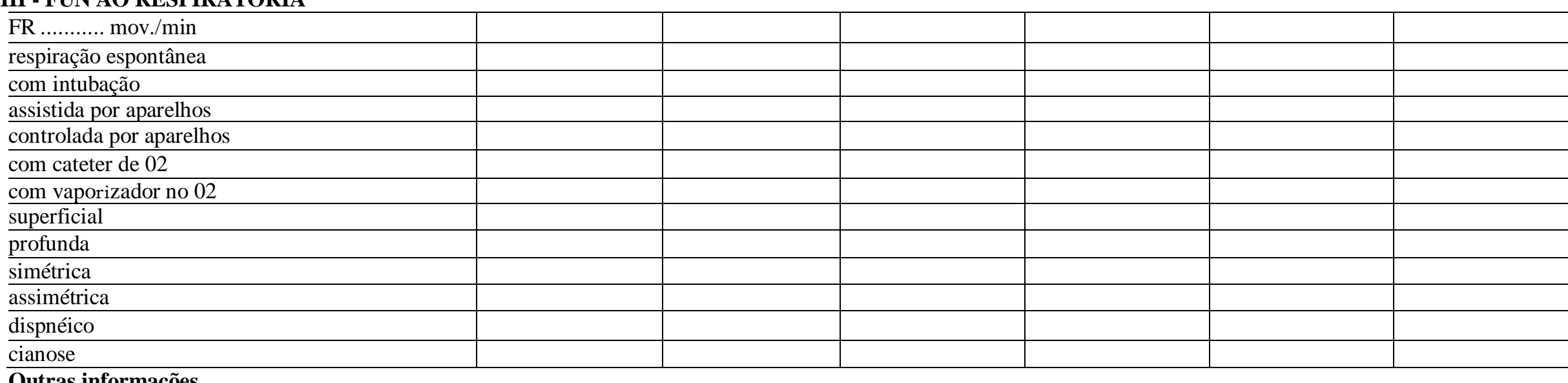

Outras informações 
FC........... bat./min.

características do pulso: cheio

\begin{tabular}{l|l}
\hline \multicolumn{1}{c|}{ forte } & \\
\hline faracterísticas do pulso: cheio & \\
\hline arrítmico & \\
\hline rítmico & \\
\hline PA........ mmHg & \\
\hline presença de edema - localizar & \\
\hline PVC......cm H20 & \\
\hline $\begin{array}{l}\text { hidratação - Presenças de soros especificar } \\
\text { tipo, punção e gotejamento }\end{array}$ & \\
\hline verbaliza sede & \\
\hline mucosas e pele seca & \\
\hline
\end{tabular}

\begin{tabular}{|l|l|}
\hline & \\
\hline & \\
\hline & \\
\hline & \\
\hline & \\
\hline & \\
\hline & \\
\hline & \\
\hline
\end{tabular}

\begin{tabular}{|l|l|}
\hline & \\
\hline & \\
\hline & \\
\hline & \\
\hline & \\
\hline & \\
\hline & \\
\hline & \\
\hline
\end{tabular}

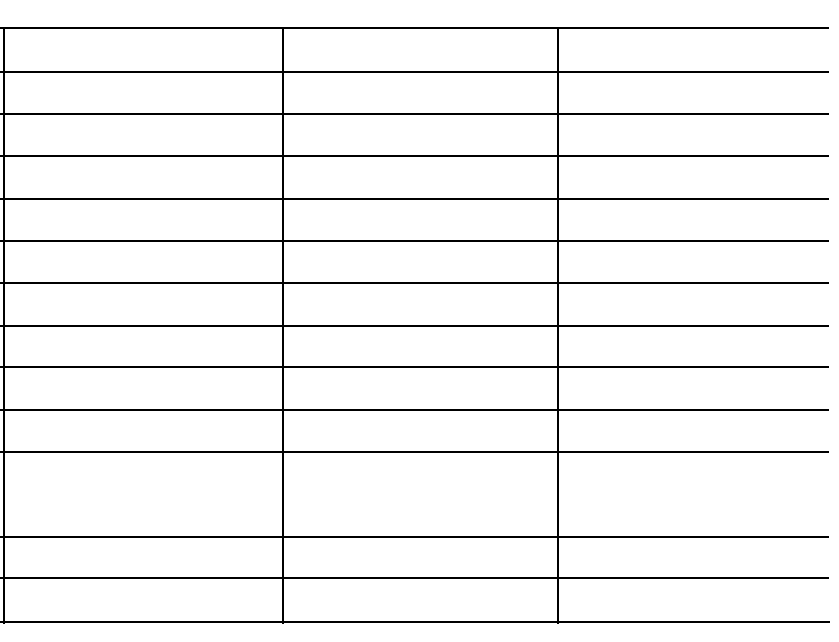

Outras informações

\section{V - FUNCÃO RENAL}

sonda vesical

diurese espontânea

volume urinário horário

Outras informações 
Tempo de permanência

VI - FUNCÃO TERMORREGULADORA

temperatura - especificar

apresenta tremores

Outras informações

\section{VII - NÍVEL DE CONSCIÊNCIA}

\section{sonolento}

confuso

alerta

orientado no tempo e espaco

agitação

expressa tranqüilidade

verbaliza dor

verbaliza desconforto

Outras informações

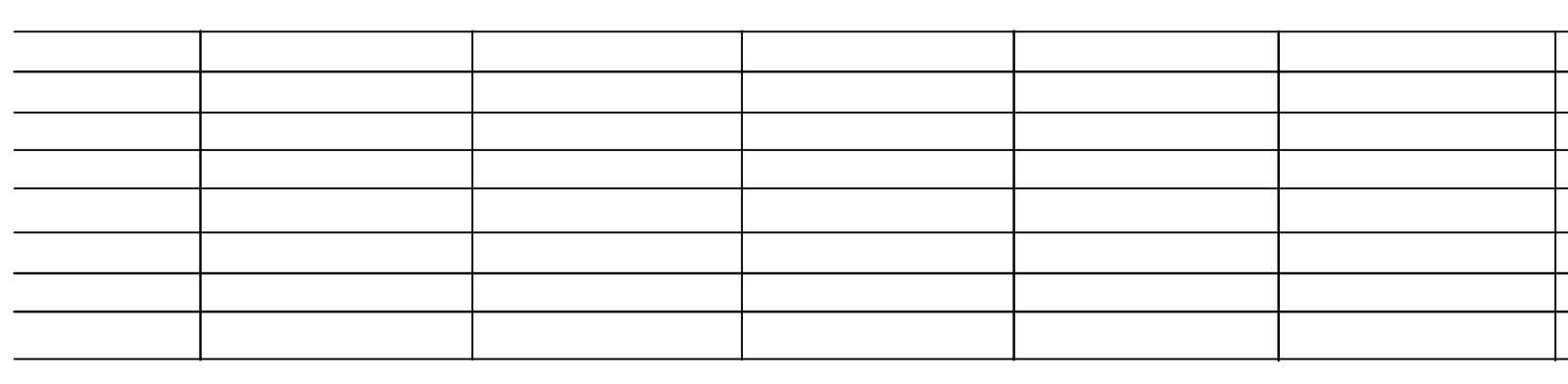

$$
\text { - }
$$


Tempo de permanência

FNCÕES DIGESTIVA E INTESTINAL

presença de sondas - localizaç ao

náuseas

vômitos

distensão abdomina

evacuações

Outras informações

\begin{tabular}{|l|l|l|l|l|l} 
& & & & & \\
& & & & & \\
\\
\hline
\end{tabular}

\section{IX - INTEGRIDADE DA PELE}

especificar estado da cicatriz e curativo cirúrgico,

presença de drenos e sangramentos, e outras alterações

\section{Outras informações}

\section{X - EQUILÍBRIO EMOCIONAL}

choro

verbaliza medo

inquietação

excitação tensão facial

voz tremula

tagarelice

Outras informações

\begin{tabular}{l|l|l|l|l|l|l}
\hline & & & & & & \\
\hline \\
\hline
\end{tabular}

Resultados de exames laboratoriais: - especificar

DATA: I / ASSINATURA 